\title{
THE EMERGENCE OF ADJACENCY PAIRS: NO EVIDENCE IS BETTER THAN NEGATIVE EVIDENCE
}

\author{
GREGORY MILLS \\ g.j.mills@rug.nl \\ Centre for Language and Cognition, University of Groningen, Netherlands
}

When participants use dialogue in joint activities, they rapidly converge on idiosyncratic referring conventions. Convergence is inherently interactive, relying on participants providing each other with both positive and negative evidence of understanding (Clark, 1996; Healey, 2007).

In addition to securing reference, interlocutors also need to coordinate on the timing and sequencing of their contributions. Dialogue is replete with procedural expressions that establish who performs which action, when the action should be performed, and how initiation and completion of the action should be signaled, e.g. "when I've done $x$, do $y$ "; "wait a moment before doing $y$ "; "let's start again, but this time you do y"; "hold on I need to tell you x first"; "do $x$ and then tell me when you're done"; "you do x and then I do y".

Recent work has demonstrated that participants rapidly establish new temporal expressions (Verhoef et al, 2016). Moreover, when temporal expressions are used interactively by participants to coordinate their actions with those of their partner, these expressions rapidly become conventionalized within novel adjacency pairs (Schegloff, 2007; Mills, 2014; Fusaroli et al., 2014). However, it is currently unclear how the central dialogue mechanisms of positive and negative feedback and alignment contribute towards conventionalization.

To investigate how procedural coordination develops, we report a computermediated "alien language" task which prevents participants from using natural language by restricting typing to a limited set of keys. However, in contrast to canonical alien language tasks which elicit referring expressions for describing referents, this task elicits procedural expressions for performing sequences of actions. 
For example, on a typical trial, the task of the Director might be to instruct the Matcher that they have to perform the following 5-step sequence:

(1) Matcher has to press $\boldsymbol{A}$;

(2) Matcher has to press $\boldsymbol{S}$

(3) Director and Matcher have to press $\boldsymbol{F}$ simultaneously

(4) Director has to press $\boldsymbol{J}$ at the same time as Matcher presses D

(5) Matcher has to press $\boldsymbol{D}$

On each trial, the target sequence (i.e. A, S, F, J, D), valence (whether both perform the action together or only one participant performs the action), and congruence (whether both perform the same or different action) are determined randomly by the server. This presents participants with the recurring procedural coordination problem of communicating and then successfully performing a wide variety of sequences of actions, without using natural language. In order to test the putative role of positive and negative feedback on how coordination develops, dyads were assigned to one of 4 conditions:

(1) Positive feedback: participants could send $\mathbf{Y}$ for yes

(2) Negative feedback: participants could send $\mathbf{N}$ for no

(3) Positive and Negative feedback: participants could send both $\mathbf{Y}$ and $\mathbf{N}$

(4) No feedback: participants were blocked from sending $\mathbf{Y}$ or $\mathbf{N}$

Participants who could provide both positive and negative feedback correctly solved more trials in shorter time, confirming the basic predictions of the grounding model (Clark, 1996). Surprisingly, participants who could signal negative evidence of understanding with " $\mathbf{N}$ " performed worse than participants who were blocked from providing any feedback. We argue this pattern is due to the intrinsic ambiguity of "no": it signals but does not diagnose the problem, and gives no indication what the next relevant action should be. By contrast, the doubly-blocked participants are forced ab-initio to develop new, and consequently more robust, routines for establishing and sustaining procedural coordination.

All four conditions showed equal levels of alignment, but closer inspection showed that, overall, participants aligned more in unsuccessful trials, contradicting the interactive alignment model (Pickering and Garrod, 2009). We argue this is due to participants using alignment as a repair strategy: if participants know the next action, they perform that next action (Clark 1996). However, when participants do not understand the contributions of their partner, one of the default strategies is to repeat the actions of the interlocutor in order to establish a basic level of coordination that can then serve as the scaffolding for coordinating on more complex and complementary adjacency pairs. 


\section{References}

Clark, H. H. (1996). Using language. Cambridge university press.

Fusaroli, R., Rączaszek-Leonardi, J., \& Tylén, K. (2014). Dialog as interpersonal synergy. New Ideas in Psychology, 32, 147-157.

Garrod, S., \& Pickering, M. J. (2009). Joint action, interactive alignment, and dialog. Topics in Cognitive Science, 1(2), 292-304.

Healey, P. G., Swoboda, N., Umata, I., \& King, J. (2007). Graphical language games: Interactional constraints on representational form. Cognitive Science, 31(2), 285-309.

Mills, G. (2011, January). The emergence of procedural conventions in dialogue. In Proceedings of the Cognitive Science Society (Vol. 33, No. 33).

Mills, G. J. (2014). Dialogue in joint activity: complementarity, convergence and conventionalization. New ideas in psychology, 32, 158-173.

Schegloff, E. A. (2007). Sequence organization in interaction: Volume 1: A primer in conversation analysis (Vol. 1). Cambridge University Press.

Verhoef, T., Walker, E. \& Marghetis, T. (2016) Cognitive biases and social coordination in the emergence of temporal language. Proceedings of the 38th Annual Conference of the Cognitive Science Society. (pp. 2615-2620) Austin,TX: Cognitive Science Society. 